\title{
Identification of Differentially Expressed Genes and Pathways for Abdominal Fat Deposition in Ovariectomized and Sham-Operated Chickens
}

\author{
Xiaopeng Mu ${ }^{1,2,3,+}$, Xiaoyan Cui ${ }^{2,3,4,+}$, Ranran Liu ${ }^{2,3}$, Qinghe $\mathrm{Li}^{2,3}$, Maiqing Zheng ${ }^{2,3}$, \\ Guiping Zhao ${ }^{2,3}$, Changrong Ge ${ }^{5}$, Jie Wen ${ }^{2,3}$, Yaodong $\mathrm{Hu}^{1, *}$ and Huanxian Cui ${ }^{2,3, *}$ \\ 1 Farm Animal Genetic Resources Exploration and Innovation Key Laboratory of Sichuan Province, \\ Sichuan Agricultural University, Chengdu Campus, Chengdu 611130, China; xiaopengmu07@126.com \\ 2 Institute of Animal Sciences, Chinese Academy of Agricultural Sciences, Beijing 100193, China; \\ cxyan813@163.com (X.C.); liuranran112@126.com (R.L.); qli2014@126.com (Q.L.); \\ wheatzheng@163.com (M.Z.); zhaoguiping@caas.cn (G.Z.); wenjie@caas.cn (J.W.) \\ 3 State Key Laboratory of Animal Nutrition, Beijing 100193, China \\ 4 Institute of Animal Science, Guangdong Academy of Agricultural Sciences, State Key Laboratory \\ of Livestock and Poultry Breeding, Key Laboratory of Animal Nutrition and Feed Science in South China \\ of Ministry of Agriculture, Guangdong Public Laboratory of Animal Breeding and Nutrition, Guangdong \\ Key Laboratory of Animal Breeding and Nutrition, Guangzhou 510640, China \\ 5 Yunnan Provincial Key Laboratory of Animal Nutrition and Feed, Yunnan Agricultural University, \\ Kunming 650201, China; gcrzal@126.com \\ * Correspondence: 13982@sicau.edu.cn (Y.H.); cuihuanxian78@126.com (H.C.); Tel.: +86-10-62816018 (H.C.); \\ Fax: 86-10-62816019 (H.C.) \\ + These authors contributed equally to this work.
}

Received: 4 December 2018; Accepted: 13 February 2019; Published: 18 February 2019

Abstract: Ovariectomy results in improved meat quality (growth rate, tenderness, and flavor) of broilers. However, some negative effects increased (abdominal fat (AF) deposition, low feed conversion, etc.) have also been reported. In this study, the gene expression profiles of AF tissue in ovariectomized and sham-operated chickens were determined to identify differentially expressed genes (DEGs) and pathways to explore the molecular mechanisms underlying AF accumulation. Comparing the ovariectomized group and the sham-operated group, the abdominal fat weight (AFW) and abdominal fat percentage (AFP) were increased significantly $(p<0.05)$ at 14 and 19 weeks after ovariectomy. According to the gene expression profiling analysis, 108 DEGs of fat metabolism were screened from 1461 DEGs. Among them, ABCA1, ABCACA, LPL, CREB1, PNPLA2, which are involved in glycerolipid—or steroid—associated biological processes, and the hormone receptor genes, ESR1 and $P R L R$, were down-regulated significantly in the ovariectomized group compared to the sham-operated group ( $p<0.05$ ). Conversely, CETP, DGAT2, DHCR24, HSD17B7 and MSMO1, were significantly up-regulated $(p<0.05)$ after ovariectomy. Based on the DEGs, the glycerolipid metabolism, steroid biosynthesis, and other signaling pathways (MAPK, TGF- $\beta$, and adhesion pathways, etc.) were enriched, which may also contribute to the regulation of AF deposition. Our data suggest that AF deposition was significantly increased in ovariectomized chickens by the down-regulation of the decomposition genes of glycerolipid metabolism, which inhibits AF degradation, and the up-regulation of steroid biosynthesis genes, which increases fat accumulation. These findings provide new insights into the molecular mechanisms of fat deposition in the ovariectomized chickens.

Keywords: chicken; abdominal fat; steroid biosynthesis; gene expression profiling; differentially expressed genes; glycerolipid metabolism 


\section{Introduction}

Ovariectomy is the removal of the ovaries of a female animal using a technique known as ovariectomization. After the ovariectomy, production performance, slaughter performance, blood biochemistry, fat metabolism, bone development, immunity, and other characteristics can undergo significant changes [1,2], which is widely used in production. For example, a previous study showed that abdominal fat (AF) deposition increases significantly after ovariectomy or caponization [3]. In general, fat deposition occurs in subcutaneous tissues, muscles, and the abdomen in chickens. The AF tissue is a reliable parameter for judging total body fat content because it is linked directly to total body fat content in avian species $[4,5]$ It is crucial in poultry because it grows faster compared with other fat tissues [6], and has a higher fat content, accounting for approximately $30 \%$ of the mass of major fat depots [7]. Reducing AF content is an urgent problem to be solved.

Adipocytes are derived from mesenchymal stem cells, which differentiate into adipoblasts, preadipocytes, and mature adipocytes. After birth, AF deposition is increased not by an increase in the number of adipocytes, but by an increase in the volume [8]. The main sources of fat in chickens are the intestinal absorption of fat from food and the de novo synthesis in the liver [9]. As energy storage in the body, a large amount of biosynthesized or absorbed lipids is transported and stored as AF, which eventually leads to degradation in meat quality as fat accumulation becomes extreme.

Although there are some available studies on the regulatory mechanisms of AF deposition in chickens of different breeds or ages [7], contrastive analyses between ovariectomized and sham-operated chickens are scarce. The purpose of this study was to explore the molecular regulatory mechanism of AF deposition in Beijing-You chickens at 14 weeks after ovariectomy. Gene expression profiling was used to identify candidate genes and related signaling pathways. The present findings provide a theoretical basis for producing higher quality chicken meat and for improving production efficiency.

\section{Materials and Methods}

\subsection{Ethics Statement}

This study was conducted in accordance with the Guidelines for Experimental Animals established by the Ministry of Science and Technology (Beijing, China). The protocol was approved by the Science Research Department (in charge of animal welfare issues) of the Institute of Animal Sciences (1 December 2013), Chinese Academy of Agricultural Sciences (Beijing, China) (No. IAS20131201).

\subsection{Animals and Treatments}

The study was conducted in accordance with the guidelines for experimental animals developed by the Ministry of Science and Technology of China (Beijing, China). Animal experiments were approved by the Science Research Department (in charge of welfare issues) at the Institute of Animal Sciences, Chinese Academy of Agricultural Sciences (CAAS) (Beijing, China). The Beijing-You (BJ-Y) chicken is a unique local breed in China, with high flavor characteristics. In this study, 80 female BJ-Y chickens with the same genetic background and similar weight were randomly distributed into two groups. The ovariectomy procedure was performed in accordance with previously described method in one of the two groups at three weeks [10], while the remaining group underwent a sham-operation (sham-operated group). Feed and water were withheld for $12 \mathrm{~h}$ before the operations. The incision site was sterilized with veterinary external disinfectant. A one centimeter lateral incision was made at the second-to-last rib, then, the ovaries were removed aseptically. The birds were raised in an environmentally-controlled room, in three-story step cages. Basal diets were formulated based on the National Resource Council (1994) requirements and the Feeding Standards of Chickens established by the Ministry of Agriculture, Beijing, China (2004).

Under carbon dioxide anesthesia, 10, 20, 10 chickens were euthanized by severing the carotid artery at 10,14, 19 weeks after the ovariectomy or sham-operations in each group, respectively. 
After slaughter, the AF was dissected in the same area for all chickens. The AF samples were weighed, snap-frozen in liquid nitrogen, and stored at $-80^{\circ} \mathrm{C}$ until use. The remaining AF tissues were removed and weighed, which included the AF pad and fat around the gizzard. The AF weight (AFW) was recorded, and the AF percentage (AFP) was calculated (mass of AF as a percentage of the live weight).

\subsection{RNA Extraction and Identification}

Total RNA was isolated from AF tissues of chickens, at 14 weeks after ovariectomy or sham-operation, using the Trizol reagent (DP419) according to the manufacturer's protocol. The quality of RNA was detected by $1 \%$ gel electrophoresis, and RNA concentration was determined by NANODROP2000 spectrophotometer (Thermo Scientific, Hudson, DE, USA). The OD260/280 values of all samples were limited to the range of 1.8 to 2.0. The RNA samples were subsequently used for gene expression profiling.

\subsection{Gene Expression Profiling and Data Analysis}

Based on ultra-high-throughput sequencing (HiSeq2500; Illumina, San Diego, CA, USA), gene expression profiling was undertaken by Berry Genomics (Beijing, China). Raw data were converted to FASTQ files using bcl2fastq (Illumina). Clean reads were generated by removing reads with adapter and low-quality sequences and mapped to the reference chicken genome and genes (Gallus gallus, Galgal4; available at https: / / www.ncbi.nlm.nih.gov/assembly/GCF_000002315.3) using TopHat 1.3.2 (https://ccb.jhu.edu/software/tophat). Gene expression levels were calculated using the RPKM method, as described by Mortazavi et al. [11]. Differentially expressed genes (DEGs) between the ovariectomized and sham-operated groups were analyzed using the edgeR R package. DEGs were screened by the following criteria: $\mid \log 2 \mathrm{FCl} \geq 0.58$, with $p<0.05$. Based on the DEGs, clustering analysis was performed in each sample by the pheatmap package of R software. Hierarchical clustering was performed on both rows and columns, and the resulting dendrogram was saved as an image file. GO (Gene Ontology) enrichment analysis was performed to identify the gene function classes and categories corresponding to the DEGs using the ClueGO plug-in and CluePedia plugin of Cytoscape (https: / / cytoscape.org /). The significance level of GO terms enrichment was set at $p<0.05$ as indicated in the Yekutieli method [12]. According to the results of GO enrichment analysis, DEGs related to abdominal adipose tissue metabolism were screened. The significantly enriched signaling pathways of DEGs were analyzed by the KEGG (Kyoto Encyclopedia of Genes and Genomes) by Kobas3.0 [13]. $p<0.05$ was considered to be indicative of statistical significance.

\section{5. qRT-PCR Analysis}

Using the same RNA samples, real-time quantitative polymerase chain reaction (qRT-PCR) was performed to confirm the results of gene expression profiling. RNA samples were reverse transcribed using TIANGEN ${ }^{\circledR}$ FastQuant RT Kit (Tiangen, Beijing, China), and specific primers were designed placing at or just outside of exon/exon junctions using Primer 5.0 software dependent on GeneBank sequences (Table S1).

Samples were amplified using the real-time PCR Detection System ABI 7500 (Applied Biosystems, Shanghai, China). The PCR mixture contained $10 \mu \mathrm{L}$ of $2 \times \mathrm{iQ}^{\mathrm{TM}}$ SYBR Green Supermix, $0.5 \mu \mathrm{L}$ (10 mmol) of each primer, and $1 \mu \mathrm{L}$ of cDNA, along with ddH2O for a total volume of $20 \mu \mathrm{L}$. After initial denaturation for $30 \mathrm{~s}$ at $95^{\circ} \mathrm{C}$, amplification was performed for 40 cycles $\left(95^{\circ} \mathrm{C}\right.$ for $5 \mathrm{~s}$ and $60{ }^{\circ} \mathrm{C}$ for $32 \mathrm{~s})$. PCR efficiency for these genes and $\beta$-actin was consistent. The comparative cycle threshold (CT) method was used to determine fold-changes in gene expression [14], with fold-changes being calculated as $2^{-\Delta \Delta C T}$. The results were expressed as the mean fold-change in gene expression from triplicate analyses, using the sham-operated group samples as the calibrators (arbitrarily assigned an expression level of 1 for each gene). Correlations between relative abundance from qRT-PCR and gene expression profiling data were also calculated. 


\subsection{Statistical Analysis}

Statistical differences between groups were evaluated using the Student's $t$-test. All computations were made suing SPSS Version 20.0 (SPSS Inc. Chicago, IL, USA). $p<0.05$ was considered significant, and data are presented as mean $\pm \mathrm{SEM}$.

\section{Results}

\subsection{Ovariectomy Accelerates AF Deposition}

Data on the AFW and AFP of ovariectomized and sham-operated chickens at 10,14, and 19 weeks after ovariectomy or sham-operations are depicted in Figure 1. The AFW and AFP of the ovariectomized group $(40.83 \mathrm{~g}, 3.96 \%)$ were significantly higher $(p<0.05)$ than those $(27.1 \mathrm{~g}, 2.95 \%)$ of the sham-operated group at 14 weeks. Similar observations were recorded at 19 weeks post-surgery (74.53 g, $6.58 \%$ vs. $39.92 \mathrm{~g}, 3.92 \%$; $p<0.05)$. There was more AF deposition in the ovariectomized group than in the sham-operated group, which suggests that AF deposition could increase after ovariectomy.

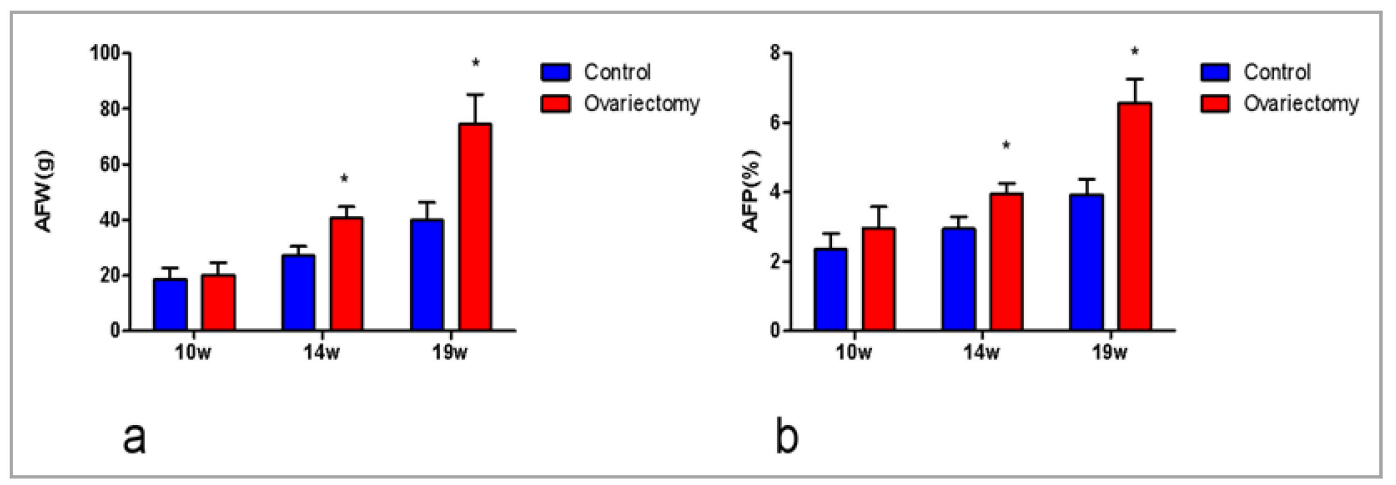

Figure 1. Abdominal fat weight (AFW) and abdominal fat percent (AFP) increase in the abdominal fat (AF) of female chickens after ovariectomy. AFW was increased in the AF of female chickens at 14 and 19 weeks after ovariectomy (a). AFP was increased in the AF of female chickens at 14 and 19 weeks after ovariectomy $(\mathbf{b})$. Data are presented as means $\pm \operatorname{SEM}\left(n=20 ;{ }^{*} p<0.05\right)$.

\subsection{Identification of DEGs Related to AF Metabolism After Ovariectomy}

Three representative chickens per group were selected at 14 weeks after ovariectomy or sham-operations, and RNA was extracted from AF tissues. Using gene expression profiling and comparing the ovariectomized group with the sham-operated group (Figure 2a), a total of 1461 DEGs ( $\mid \log 2 \mathrm{FCl} \geq 0.58$, with $p<0.05$ ) were screened, of which 1009 were down-regulated and 452 were up-regulated (Table S2). Based on these 1461 DEGs, cluster analysis was performed, and the results showed that samples of the same groups were clustered together, respectively (Figure 2b).

GO analysis was performed based on 1461 known DEGs, and the main GO terms included the following processes: Cell adhesion, regulation of the immune system, regulation of cell differentiation, transmembrane transport, and lipid metabolism (Table S3). GO enrichment analysis indicated that a large number of DEGs related to lipid metabolism (Table S4), and 12 representative DEGs were selected to validate the gene expression profiling results by qRT-PCR (Figure 3). Among them, the cholesterol homeostasis genes $A B C A 1, A B C A C A, L P L$ and $C R E B 1$ were down-regulated, but $C E T P$ was significantly up-regulated; glycerolipid metabolism gene PNPLA2 was significantly down-regulated, but DGAT2 was significantly up-regulated; steroid biosynthesis genes MSMO1, HSD17B7 and DHCR24 were significantly up-regulated; and hormone receptor genes ESR1 and PRLR were also significantly down-regulated after ovariectomy $(p<0.05)$. To confirm the accuracy of the data, the results of gene expression profiling and qRT-PCR were analyzed by Spearman rank correlation, and the fold change of gene expression between the two methods was significantly correlated (Figure 4) $(r=0.9802, p<0.05)$. 


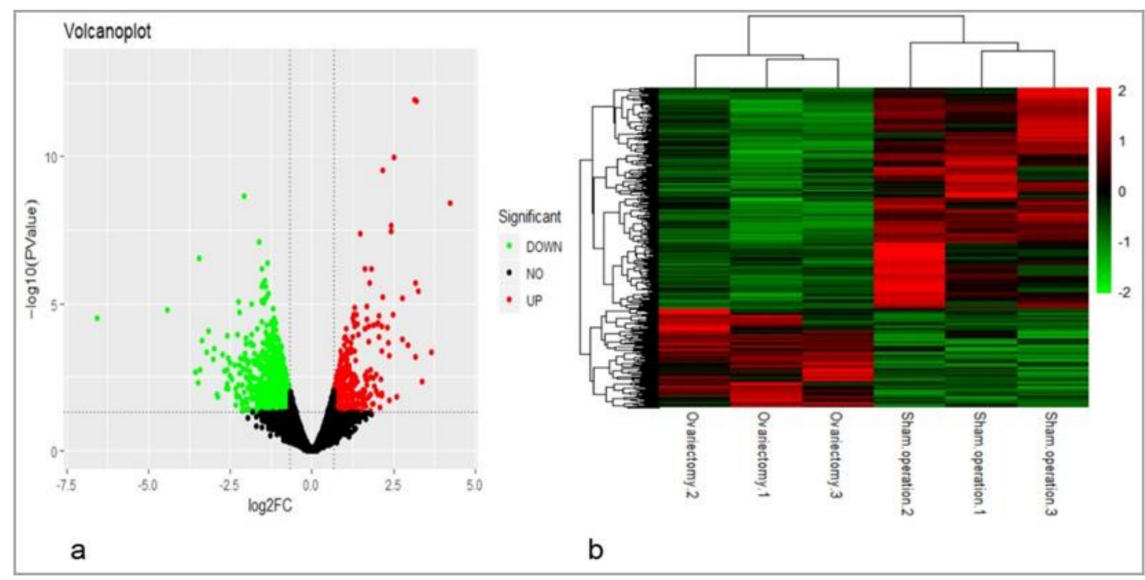

Figure 2. Volcano plot and cluster analysis of differentially expressed genes (DEGs). (a) Volcano plot. Red dots (UP) represent significantly up-regulated genes $(\log 2 \mathrm{FC} \geq 0.58, p<0.05)$; green dots $(\mathrm{DOWN})$ represent significantly down-regulated genes $(\log 2 \mathrm{FC} \leq-0.58, p<0.05)$; black dots (NO) represent DEGs below the level of significance; (b) based on 1461 known DEGs in the AF tissue of the ovariectomized and sham-operated groups at 14 weeks post-surgery, cluster analysis was performed. The results show that the data in the gene expression profiling of chickens in same group were closely related.

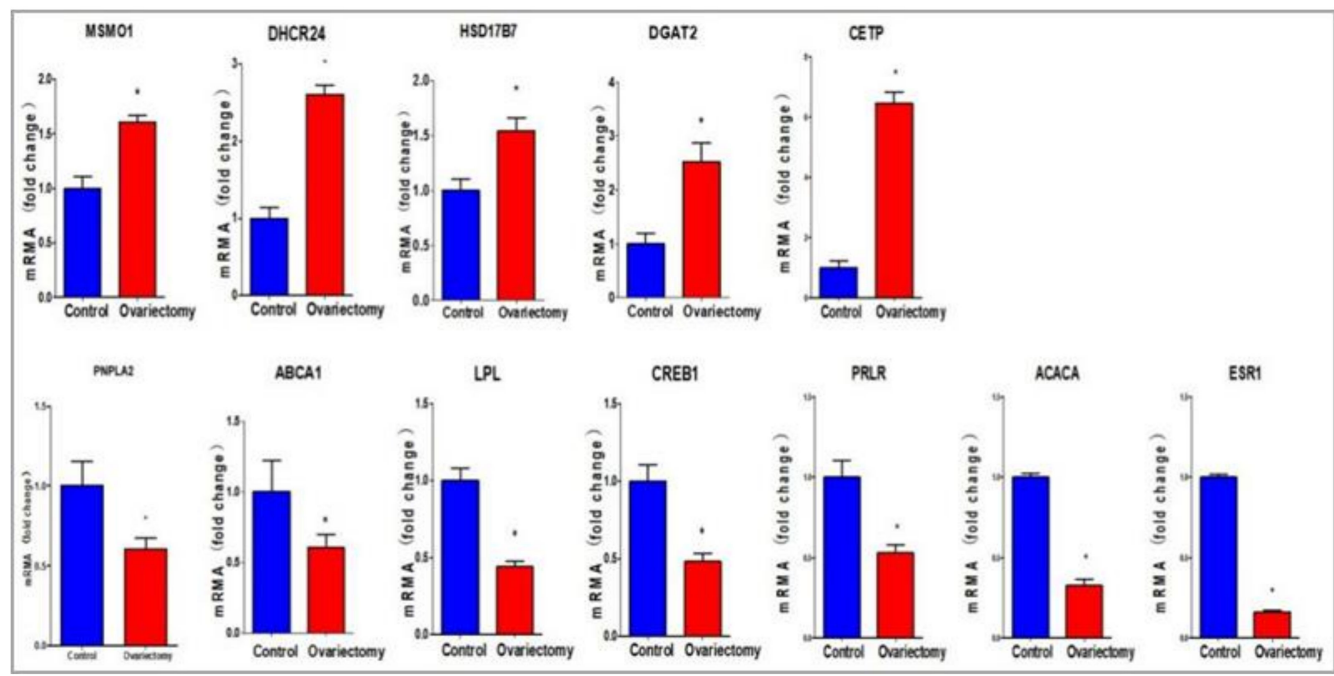

Figure 3. Real-time quantitative polymerase chain reaction (qRT-PCR) verification of DEGs detected by gene expression profiling. The expression levels of DEGs related to lipid metabolism determined by qRT-PCR in the ovariectomized and sham-operated groups. These genes were involved in glycerolipid metabolism, sterol biosynthesis, and fatty acid biosynthesis, among others. Each of these DEGs were up-regulated or down-regulated significantly $(p<0.05)$ in the ovariectomized group compared with the sham-operated group. 


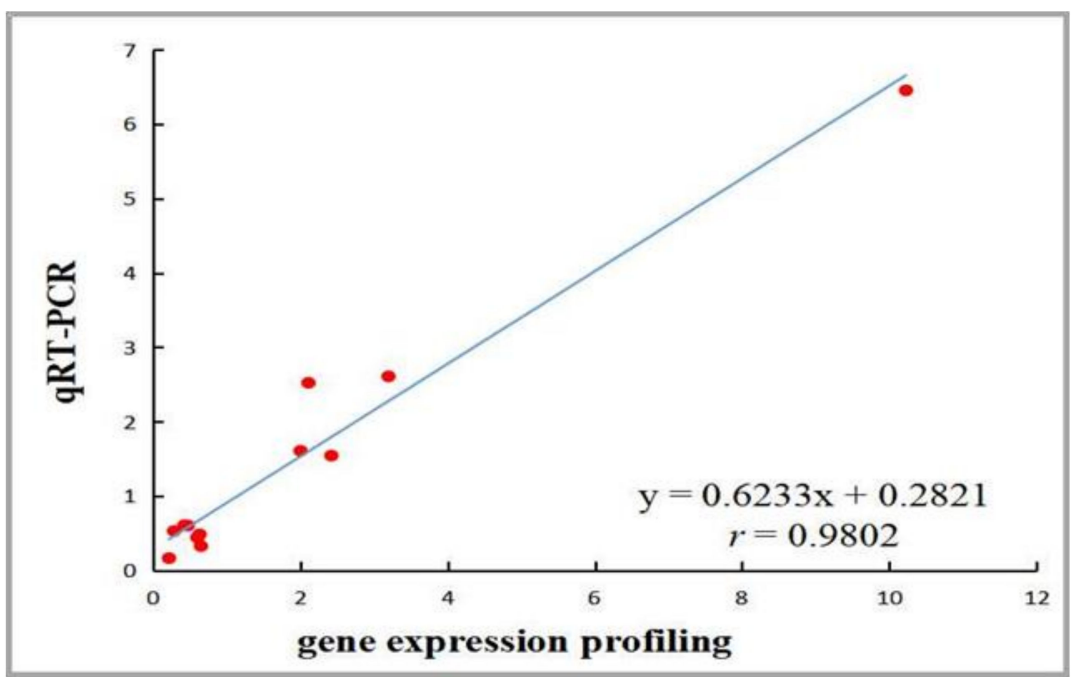

Figure 4. Correlation analysis of gene expression profiling and qRT-PCR results. The correlation between gene expression profiling and qRT-PCR data was analyzed by Spearman rank correlation in the ovariectomized and sham-operated groups. A high correlation coefficient $(r=0.9802, p<0.05)$ was detected, which indicates that the gene expression profiling data are reliable.

\subsection{Pathways and Regulatory Network for AF in Chickens After Ovariectomy}

Based on 1461 known DEGs, a KEGG pathway analysis was performed and 17 pathways were found to be significantly enriched $(p<0.05)$ (Figure 5$)$. As expected, some pathways related to lipid metabolism were screened (e.g., glycerolipid metabolism, steroid biosynthesis, TGF-signaling pathway, MAPK signaling pathways, etc.). In addition, some cell adhesion signaling pathways (focal adhesion, adherens junction and regulation of actin cytoskeleton) were also significantly enriched $(p<0.05)$.

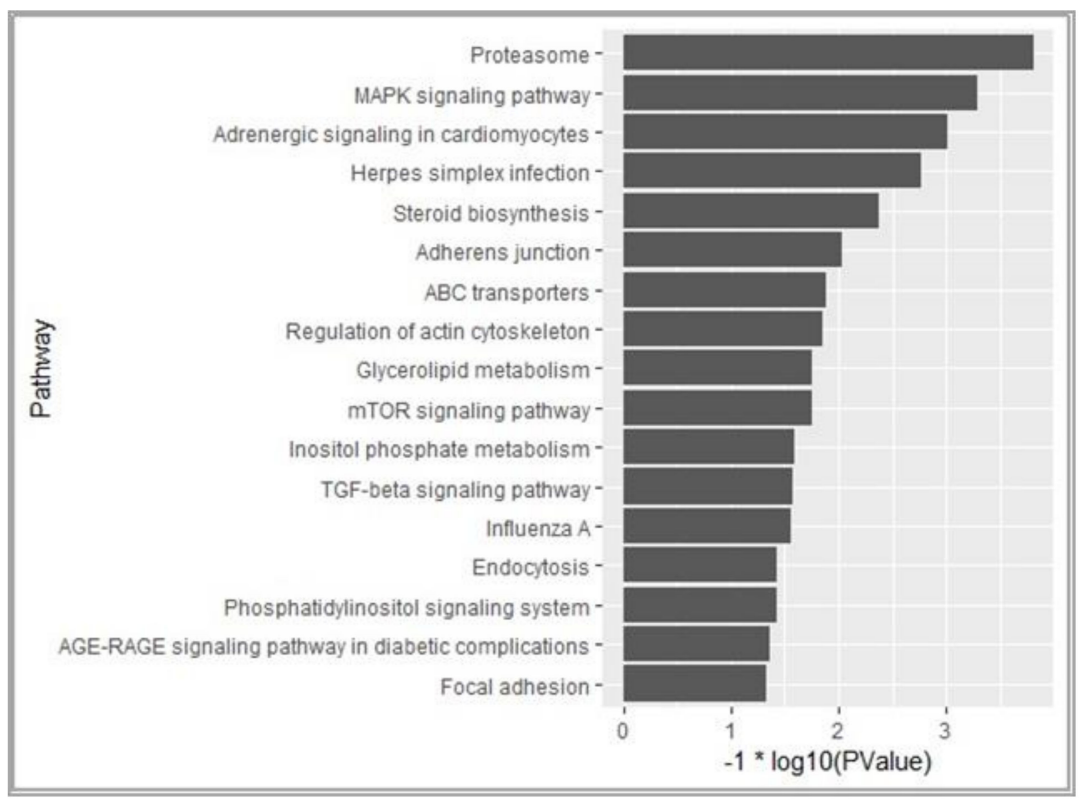

Figure 5. Enriched pathways based on the 1461 DEGs. KEGG (Kyoto Encyclopedia of Genes and Genomes) pathway analysis of DEGs showed that various fat metabolism pathways (glycerolipid metabolism, steroid biosynthesis etc.) were enriched $(p<0.05)$. 


\section{Discussion}

\subsection{Differences in AF Deposition between the Ovariectomized and Sham-Operated Groups}

Ovariectomy plays an important role in fat deposition. Studies have shown that the weight of AF in goats was significantly increased after ovariectomy [15]. AF deposition was also found to increase after ovariectomy in mice compared to a control group [16,17], and ovariectomy may stimulate adipocyte hypertrophy, therefore promoting obesity [18]. All these findings are consistent with our research which shows that AFW and AFP were up-regulated significantly in ovariectomized chickens at 14- and 19-weeks post-surgery, with the gap increasing over time.

\subsection{The Validation of Gene Expression Profiling and Identification of DEGs Related to AF Deposition}

High quality total RNA samples were obtained and used for gene expression profiling. To validate the obtained results, cluster analysis was performed. The samples of the ovariectomized group and sham-operated group clustered closely, respectively. Furthermore, 12 representative genes were screened from 108 known DEGs related to fat metabolism, and qRT-PCR analysis was performed to validate the gene expression profiling. The fold changes in gene expression levels were used to correlate the results of RNA-seq and qRT-PCR, which revealed a significant correlation $(p<0.05)$. These results confirmed the reliability of the obtained gene expression profiling data.

\subsection{Key Genes Related to the Metabolism of AF}

Based on the 1461 known DEGs, GO terms analysis was performed, the main GO terms included the following processes: Cell adhesion, regulation of the immune system, regulation of cell differentiation, transmembrane transport, and lipid metabolism. Among them, the gene expression levels of $A B C A 1, A B C A C A$, and $L P L$ were significantly down-regulated in the ovariectomized compared to the sham-operated group. As a cholesterol efflux pump in the cellular lipid removal pathway, $A B C A 1$ has an important function in cholesterol efflux. Consequently, lower $A B C A 1$ expression could reduce lipid efflux, leading to lipid accumulation [19]. ACACA is important for malonyl CoA, which catalyzes the synthesis of long-chain fatty acids [20]. The LPL gene encodes lipoprotein lipase, which acts as the rate-limiting step in the hydrolysis of triglycerides, negatively correlating with fat deposition [21,22]. Similarly, PNPLA2, CREB1, ESR1, and PRLR had lower expression in AF of the ovariectomized group compared to the sham-operated group. PNPLA2 encodes the key enzyme of triglyceride hydrolysis, participating in the regulation of the first step in triglyceride hydrolysis [23]. CREB1 is up-regulated by P38 stimulation, and causes a decrease in steroidogenesis [24]. Estrogen are well-known regulators of fat distribution and deposition, with research showing lower ESR1 expression in adipocytes of obese compared to non-obese premenopausal women [24,25]. PRLR has a positive contribution to triglyceride accumulation by increasing expression in the liver [25]. These results suggest that these genes could increase AF deposition by decreasing fat degradation.

Some genes (MSMO1, DHCR24, HSD17B7) that participate in the steroid biosynthesis process were up-regulated in the ovariectomized group compared to the sham-operated group. The MSMO1 gene was expressed in diabetic animal models and shown to play key roles in cholesterol biosynthesis, energy metabolism, obesity, and dyslipidemia regulation [26,27]. DHCR24 encodes the enzyme involved in the final step of cholesterol synthesis, catalyzing the synthesis of cholesterol from chain sterols [28]. HSD17B7 encodes an enzyme that functions both as a 17- $\beta$-hydroxysteroid dehydrogenase in the biosynthesis of sex steroids and as a 3-ketosteroid reductase in the biosynthesis of cholesterol [29]. These results suggest that increasing expression of these genes could contribute to the increase in AF deposition after ovariectomy.

In addition, the expression levels of DGAT2 and CETP were significantly higher in AF of the ovariectomized group than in the AF of the sham-operated group. DGAT2 is critical for adipose tissue formation, catalyzing the final step in triacylglycerol synthesis. Overexpression of DGAT2 in 
mammalian HEK293 cells can significantly increase triglyceride synthesis [30,31]. CETP is involved in the transfer of neutral lipids (cholesteryl ester and triglycerides) and in the positively regulated phospholipid accumulation in chickens [32]. These results reveal that DGAT2 and CETP could increase AF deposition in ovariectomized chickens.

\subsection{Signaling Pathways Related to Lipid Metabolism in AF after Ovariectomy}

The regulation of AF deposition is possibly a result of complex interactions between multiple pathways. Based on the screened DEGs, several well-known pathways related to lipid metabolism were found to be enriched (i.e., glycerolipid metabolism, steroid biosynthesis, TGF- $\beta$ signaling pathway, MAPK signaling pathway, etc.). In addition, some cell adhesion signaling pathways (focal adhesion, adherens junction and regulation of actin cytoskeleton) were also enriched significantly $(p<0.05)$, which was consistent with the results of a previous study [32].

Glycerolipid metabolism, regulated by $D G K H, D G K B, D G K E, D G K D, P N P L A 2, P P A P 2 B$, ALDH9A1, GPAM, LPL, AGPAT4, DGAT2, has been reported to be significantly associated with fat metabolism [33]. According to previous research, DGKE plays a prominent role in enriching inositol phospholipids with unsaturated fatty acids and lipid synthesis [34]. DGKD knockout leads to a decrease in fatty acid synthesis-related enzymes, as well as in the levels of many lipids within the cell [35]. In addition, some steroid biosynthesis genes (MSMO1, CYP51A1, HSD17B7, LIPA and DHCR24) were also significantly up-regulated. As well known, the TGF- $\beta$ and MAPK signaling pathways participate in the regulation of fat metabolism [36-38]. Interestingly, several studies suggest that cell adhesion-related pathways are involved in lipid deposition $[3,38]$. Three common DEGs (VCL, ACTB and $R H O A$ ) are shared by these pathways. Several DEGs of adhesion-related pathways (FGF12, FGFR2, TGFBR2) are involved in the MAPK signaling pathway [39]. Studies have shown that MAPK activation results in the disruption of tight junctions, whereas the inhibition of MAPK activation prevents this process [40]. Additionally, P38 was shown to negatively regulate steroidogenesis by increasing the expression of $C R E B$ [41]. These findings collectively suggest that multiple adhesion-related signaling pathways could interact and participate in the regulation of AF deposition.

Based on these pathways, a possible molecular regulatory network managing AF deposition in chickens was constructed (Figure 6), suggesting that the increased AF deposition may be the result of multiple signaling pathways acting together.

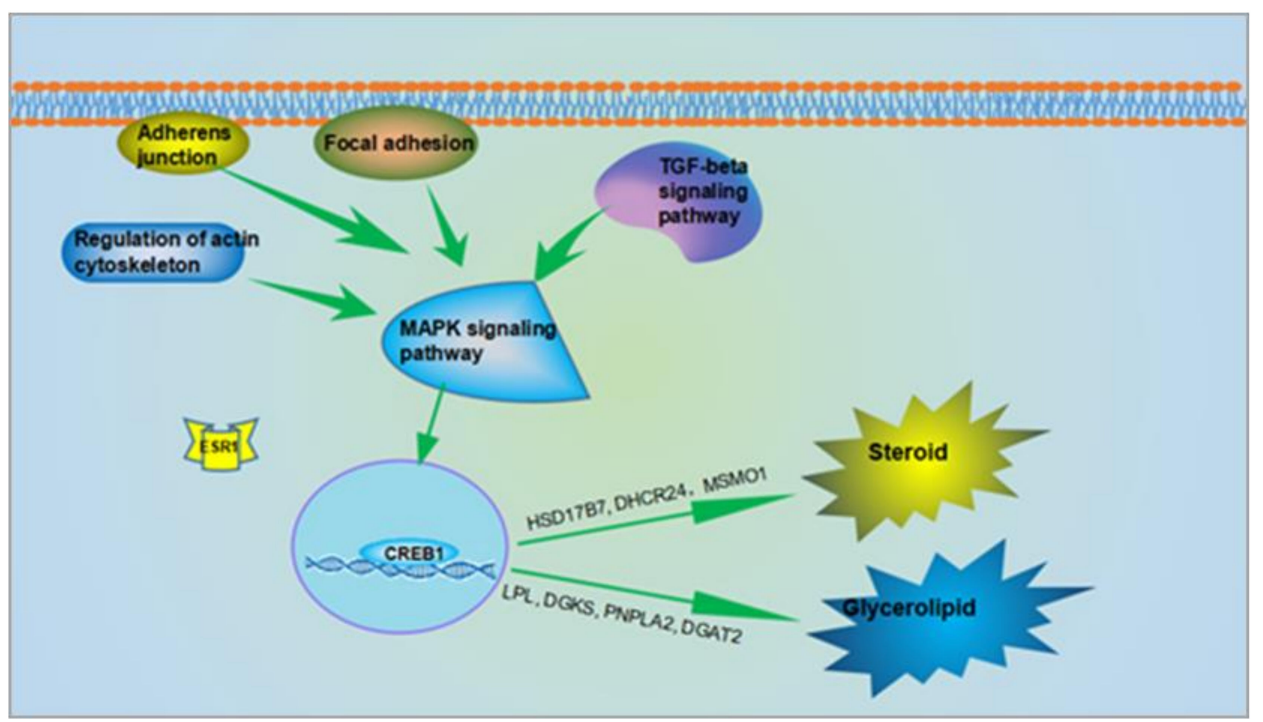

Figure 6. The potential regulatory network mediating lipid deposition in ovariectomized chickens. The AF deposition regulatory network may include: Glycerolipid metabolism, steroid metabolism, MAPK, TGF- $\beta$, and cell junctions (cell adhesion, adherens junctions, regulation of actin cytoskeleton) pathways. 


\section{Conclusions}

In this study, our data suggests that $\mathrm{AF}$ deposition was significantly increased in ovariectomized chickens by the down-regulation of the decomposition genes (ABCA1, ABCACA, CREB1, PNPLA2, ESR1, PRLR, LPL) of glycerolipid metabolism, which inhibits AF degradation, and the up-regulation of steroid biosynthesis genes (CETP, DGAT2, DHCR24, HSD17B7, MSMO1), which increases fat accumulation. They work together to increase the deposition of AF. Additionally, cell adhesions, TGF- $\beta$ and MAPK signaling pathways were also found, which is possibly involved in the regulation of AF deposition. These findings provide new insights into the regulation of AF deposition in the ovariectomized chickens.

Supplementary Materials: The following are available online at http:/ / www.mdpi.com/2073-4425/10/2/155/s1, Table S1: The specific qRT-PCR primers in this study (XLSX). Table S2: The screened 1461 DEGs in the study (XLSX). Table S3: The enriched the GO terms based on 1461 DEGs (XLSX). Table S4: The 108 DEG related to lipid metabolism (XLSX).

Author Contributions: X.M. and X.C. performed of the study and wrote the manuscript; R.L. Q.L, and M.Z contributed to interpretation of data; G.Z., J.W. and C.G. contributed to the design of the study; H.C. and Y.H. contributed to the design of the study and modifying the manuscript.

Funding: The research was supported by grants from the National Natural Science foundation of China (31301133, 31372305), the Agricultural Science and Technology Innovation Program (ASTIP-IAS-TS-11 and ASTIP-IAS04), the Earmarked Fund for Modern Agro-industry Technology Research System (CARS-41) and sichuan science research (2017NZ0074, 2018HH0083).

Conflicts of Interest: The authors declare no conflict of interest.

\section{References}

1. Amorim, A.; Rodrigues, S.; Pereira, E.; Valentim, R.; Teixeira, A. Effect of caponisation on physicochemical and sensory characteristics of chickens. Animal 2016, 10, 978-986. [CrossRef] [PubMed]

2. Calik, J.; Połtowicz, K.; Światkiewicz, S.; Krawczyk, J.; Nowak, J. Effect of caponization on meat quality of Greenleg Partridge cockerels. Ann. Anim. Sci. 2015, 15, 541-553. [CrossRef]

3. Cui, X.; Cui, H.; Liu, L.; Zhao, G.; Liu, R.; Li, Q.; Zheng, M.; Wen, J. Decreased testosterone levels after caponization leads to abdominal fat deposition in chickens. BMC Genom. 2018, 19, 344. [CrossRef] [PubMed]

4. Becker, W.A.; Spencer, J.V.; Mirosh, L.W.; Verstrate, J.A. Prediction of fat and fat free live weight in broiler chickens using backskin fat, abdominal fat, and live body weight. Poult. Sci. 1979, 58, 835-842. [CrossRef]

5. Thomas, V.G.; Mainguy, S.K.; Prevett, J.P. Predicting fat content of geese from abdominal fat weight. J. Wildl. Manag. 1983, 47, 1115-1119. [CrossRef]

6. Butterwith, S.C. Contribution of lipoprotein lipase activity to the differential growth of three adipose tissue depots in young broiler chickens. Br. Poult. Sci. 1989, 30, 927-933. [CrossRef]

7. Resnyk, C.W.; Carré, W.; Wang, X.; Porter, T.E.; Simon, J.; Bihan-Duval, E.L.; Duclos, M.J.; Aggrey, S.E.; Cogburn, L.A. Transcriptional analysis of abdominal fat in chickens divergently selected on bodyweight at two ages reveals novel mechanisms controlling adiposity: Validating visceral adipose tissue as a dynamic endocrine and metabolic organ. BMC Genom. 2017, 18, 626. [CrossRef]

8. Leclercq, B. Adipose tissue metabolism and its control in birds. Poult. Sci. 1984, 63, 2044-2054. [CrossRef]

9. Leveille, G.A. In vitro hepatic lipogenesis in the hen and chick. Comp. Biochem. Physiol. 1969, 28, 431-435. [CrossRef]

10. Song, Y.; Silversides, F.G. The technique of orthotopic ovarian transplantation in the chicken. Poult. Sci. 2006, 85, 1104-1106. [CrossRef]

11. Mortazavi, A.; Williams, B.A.; McCue, K.; Schaeffer, L.; Wold, B. Mapping and quantifying mammalian transcriptomes by RNA-Seq. Nat. Methods 2008, 5, 621. [CrossRef] [PubMed]

12. Benjamini, Y.; Yekutieli, D. The control of the false discovery rate in multiple testing under dependency. Ann. Stat. 2001, 29, 1165-1188.

13. Wu, J.; Mao, X.; Tao, C.; Luo, J.; Wei, L. KOBAS server: A web-based platform for automated annotation and pathway identification. Nucleic Acids Res. 2006, 34, W720-W724. [CrossRef] [PubMed] 
14. Livak, K.J.; Schmittgen, T.D. Analysis of relative gene expression data using real-time quantitative PCR and the $2-\Delta \Delta$ C T Method. Methods Companion Methods Enzymol. 2001, 25, 402-408. [CrossRef] [PubMed]

15. Zhang, L.; Wang, Y.; Fu, M.; Li, G.; An, N.; Li, S.; Zhou, Z. The effects of ovariectomy on meat performance and expression of GH/IGF-I in young goats. Can. J. Anim. Sci. 2014, 94, 619-626. [CrossRef]

16. Meli, R.; Pacilio, M.; Raso, G.M.; Esposito, E.; Coppola, A.; Nasti, A.; Di Carlo, C.; Nappi, C.; Di Carlo, R. Estrogen and raloxifene modulate leptin and its receptor in hypothalamus and adipose tissue from ovariectomized rats. Endocrinology 2004, 145, 3115-3121. [CrossRef]

17. Babaei, P.; Dastras, A.; Tehrani, B.S.; Roudbaneh, S.P. The effect of estrogen replacement therapy on visceral fat, serum glucose, lipid profiles and apelin level in ovariectomized rats. J. Menopausal Med. 2017, 23, 182-189. [CrossRef]

18. Wang, J.F.; Guo, Y.X.; Niu, J.Z.; Liu, J.; Wang, L.Q.; Li, P.H. Effects of Radix Puerariae flavones on liver lipid metabolism in ovariectomized rats. World J. Gastroenterol. 2004, 10, 1967-1970. [CrossRef]

19. Lee, J.; Park, Y.; Koo, S.I. ATP-binding cassette transporter A1 and HDL metabolism: Effects of fatty acids. J. Nutr. Biochem. 2012, 23, 1-7. [CrossRef]

20. Liya, Y.; Yanqiao, Z.; F Bradley, H. Sterol regulatory element-binding protein-1 interacts with the nuclear thyroid hormone receptor to enhance acetyl-CoA carboxylase- $\alpha$ transcription in hepatocytes. J. Biol. Chem. 2002, 277, 19554-19565.

21. Aaltonen, T.; Adelman, J.; Akimoto, T.; Albrow, M.G.; Alvarez, G.L.B.; Amerio, S.; Amidei, D.; Anastassov, A.; Annovi, A.; Antos, J. First measurement of $\mathrm{W}$ boson production in association with a single Charm quark in pp collisions at sqrt(s) $=1.96 \mathrm{TeV}$. Phys. Rev. Lett. 2008, 100, 091803. [CrossRef] [PubMed]

22. Goldberg, I.J.; Merkel, M. Lipoprotein lipase: Physiology, biochemistry, and molecular biology. Front. Biosci. 2001, 6, D388-D405. [PubMed]

23. Suzuki, A.; Yamaguchi, S.; Li, M.; Hara, Y.; Miyauchi, H.; Ikeda, Y.; Zhang, B.; Higashi, M.; Ikeda, Y.; Takagi, A.; et al. Tricaprin rescues myocardial abnormality in a mouse model of triglyceride deposit cardiomyovasculopathy. J. Oleo Sci. 2018. [CrossRef] [PubMed]

24. Horton, J.D.; Iichiro, S.; Shinji, I.; Yuriy, B.; Hammer, R.E. Overexpression of sterol regulatory element-binding protein-1a in mouse adipose tissue produces adipocyte hypertrophy, increased fatty acid secretion, and fatty liver. J. Biol. Chem. 2003, 278, 36652-36660. [CrossRef] [PubMed]

25. Nilsson, M.; Dahlman, I.; Rydén, M.; Nordström, E.A.; Gustafsson, J.A.; Arner, P.; Dahlman-Wright, K. Oestrogen receptor $\alpha$ gene expression levels are reduced in obese compared to normal weight females. Int. J. Obes. 2007, 31, 900. [CrossRef] [PubMed]

26. Chen, G.; Bentley, A.; Adeyemo, A.; Shriner, D.; Zhou, J.; Doumatey, A.; Huang, H.; Ramos, E.; Erdos, M.; Gerry, N. Genome-wide association study identifies novel loci association with fasting insulin and insulin resistance in African Americans. Hum. Mol. Genet. 2012, 21, 4530-4536. [CrossRef] [PubMed]

27. Lu, Y.; Dollé, M.E.T.; Imholz, S.; Slot, R.V.T.; Verschuren, W.M.M.; Wijmenga, C.; Feskens, E.J.M.; Boer, J.M.A. Multiple genetic variants along candidate pathways influence plasma high-density lipoprotein cholesterol concentrations. J. Lipid Res. 2008, 49, 2582-2589. [CrossRef]

28. Fitzpatrick, D.R.; Keeling, J.W.; Evans, M.J.; Kan, A.E.; Bell, J.E.; Porteous, M.E.; Mills, K.; Winter, R.M.; Clayton, P.T. Clinical phenotype of desmosterolosis. Am. J. Med. Genet. Part A 2010, 75, 145-152. [CrossRef]

29. Laubner, D.; MÖller, G.; Marijanovic, Z.; Adamski, J.; Gege, C.; Husen, B.; Breitling, R. Closing the gap: identification of human 3-ketosteroid reductase, the last unknown enzyme of mammalian cholesterol biosynthesis. Mol. Endocrinol. 2003, 17, 1715-1725.

30. Cases, S.; Smith, S.J.; Zheng, Y.W.; Myers, H.M.; Lear, S.R.; Sande, E.; Novak, S.; Collins, C.; Welch, C.B.; Lusis, A.J. Identification of a gene encoding an acyl CoA:diacylglycerol acyltransferase, a key enzyme in triacylglycerol synthesis. Proc. Natl. Acad. Sci. USA 1998, 95, 13018-13023. [CrossRef]

31. Mun Ock, K.; Ui, L.S.; Hyun-Jun, L.; Kwangman, C.; Hyeongki, K.; Sangku, L.; Soo Jin, O.; Sunhong, K.; Jong Soon, K.; Hyun Sun, L. Identification and validation of a selective small molecule inhibitor targeting the diacylglycerol acyltransferase 2 activity. Biol. Pharm. Bull. 2013, 36, 1167-1173.

32. Cui, H.-X.; Liu, R.-R.; Zhao, G.-P.; Zheng, M.-Q.; Chen, J.-L.; Wen, J. Identification of differentially expressed genes and pathways for intramuscular fat deposition in pectoralis major tissues of fast-and slow-growing chickens. BMC Genom. 2012, 13, 213. [CrossRef] [PubMed]

33. Zhang, P.; Reue, K. Lipin proteins and glycerolipid metabolism: Roles at the ER membrane and beyond. Biochim. Biophys. Acta (BBA)-Biomembr. 2017, 1859, 1583-1595. [CrossRef] [PubMed] 
34. Chibalin, A.V.; Leng, Y.; Vieira, E.; Krook, A.; Björnholm, M.; Long, Y.C.; Kotova, O.; Zhong, Z.; Sakane, F.; Steiler, T.; et al. Downregulation of diacylglycerol kinase delta contributes to hyperglycemia-induced insulin resistance. Cell 2008, 132, 375-386. [CrossRef]

35. Shulga, Y.V.; Loukov, D.; Ivanova, P.T.; Milne, S.B.; Myers, D.S.; Hatch, G.M.; Umeh, G.; Jalan, D.; Fullerton, M.D.; Steinberg, G.R. Diacylglycerol kinase delta promotes lipogenesis. Biochemistry 2013, 52, 7766-7776. [CrossRef] [PubMed]

36. Nam, D.; Guo, B.; Chatterjee, S.; Chen, M.H.; Nelson, D.; Yechoor, V.K.; Ma, K. The adipocyte clock controls brown adipogenesis via TGF- $\beta$ /BMP signaling pathway. J. Cell Sci. 2015, 128, 1835-1847. [CrossRef] [PubMed]

37. Huang, H.; Song, T.J.; Li, X.; Hu, L.; He, Q.; Liu, M.; Lane, M.D.; Tang, Q.Q. BMP signaling pathway is required for commitment of $\mathrm{C} 3 \mathrm{H} 10 \mathrm{~T} 1 / 2$ pluripotent stem cells to the adipocyte lineage. Proc. Natl. Acad. Sci. USA 2009, 106, 12670-12675. [CrossRef] [PubMed]

38. Peterson, T.; Sengupta, S.; Harris, T.; Carmack, A.; Kang, S.; Balderas, E.; Guertin, D.; Madden, K.; Carpenter, A.; Finck, B. mTOR complex 1 regulates lipin 1 localization to control the SREBP pathway. Cell 2011, 146, 408-420. [CrossRef]

39. Martin, G.R. The roles of FGFs in the early development of vertebrate limbs. Genes Dev. 1998, 12, 1571-1586. [CrossRef]

40. Chiou, M.J.; Wang, Y.D.; Kuo, C.M.; Chen, J.C.; Chen, J.Y. Functional analysis of mitogen-activated protein kinase-3 (MAPK3) and its regulation of the promoter region in zebrafish. DNA Cell Biol. 2007, 26, 781-790. [CrossRef]

41. Syed Kashif, Z.; Wen-Jun, S.; Stefanie, B.; Alex, B.; Mclean, M.P.; Jiahuai, H.; Davis, R.J.; Kraemer, F.B.; Salman, A. p38 MAPK regulates steroidogenesis through transcriptional repression of STAR gene. J. Mol. Endocrinol. 2014, 53, 1-16.

(C) 2019 by the authors. Licensee MDPI, Basel, Switzerland. This article is an open access article distributed under the terms and conditions of the Creative Commons Attribution (CC BY) license (http://creativecommons.org/licenses/by/4.0/). 